\title{
Study on the Efficient Methods for Chicken Eggshell windowing and the Factors Affecting the Hatchability
}

Original Article

\section{Euthor(s)}

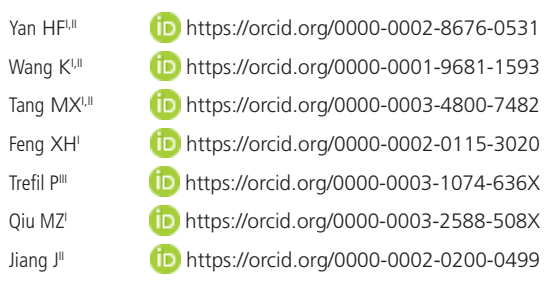

Hunan Institute of Animal and Veterinary Science, Changsha 410131, Hunan, China.

" College of Animal Science and Technology, Hunan Agricultural University, Changsha 410128, Hunan, China.

III Research Institute of Biopharmacy and Veterinary Drugs, Pohori-Chotoun, 25449 Jilove u Prague, Czech Republic)

\section{Mail Address}

Corresponding author e-mail address Haifeng Yan

Hunan Institute of Animal and Veterinary Science, Changsha 410131, Hunan, China.

Phone: 008613787293228

Email: hfyan2005@163.com

\section{-Keywords}

Air cell, blastoderm, chicken, eggshell windowing, hatchability.

\section{ABSTRACT}

In order to find out the main factors influenced the hatchability and improve the hatchability of the windowed chicken eggs at stage $X$, several experiments were made on the basis of the former patent of eggshell windowing methods on equatorial plane, such as cutting and sealing techniques, air cell recovering, laying position immediately after sealing, as well as the injection volumes into the subgerminal cavity of the blastoderm. The result showed that:1) the best sealing material combination was straw powder (SP) and instant glue (IG); 2) there was a highly positive correlation between air cell rate and hatchability; 3 ) the highest hatchability increased to $71.6 \%$ when the eggs were windowed and sealed with IG dropped firstly and then SP sprinkled, finally lay down with the blunt end upward immediately after being sealed; 4) the hatchability was significantly reduced as injection volume (DMEM) was increased $(p<0.05$ or $p<0.01)$ from $1 \mu \mathrm{L}$ to $101 \mu \mathrm{L}$, and the group of injecting $1 \mu \mathrm{L}$ was the highest (48.4\%). The hatchability and efficiency with such method of windowing, injecting, and sealing was the highest at the present time (more than 30 eggs per hour per person), and it might be broadly used in the fields of avian transgenesis, genetic resources preservation, and embryonic development model of human medicine.

\section{INTRODUCTION}

Compared with mammals, birds were deemed to be an ideal model and bioreactor for developmental biology for their shorter generation time, lower cost, higher fecundity (Stern et al., 2005). It showed that the chorioallantoic membrane (CAM) provided a flexible and lowcost in ovo strategy model and as the CAM was an extraembryonic membrane with an extensive vascular network and it was proposed as an excellent interface for xenotransplantation (Farzaneh et al.,2018). The combination of a surrogate eggshell system and PGCs (primordial germline cells) mediated transplantation technique could be a valuable tool for avian germline manipulation and production of recombinant proteins in eggs (Farzaneh et al.,2018). The chick embryo has become a research hotspot in several research fields, such as genetics, virology, cancer, cell biology (Farzaneh et al.,2018; Wang et al.,2019), as well as transgenic technology and germline modification in animal husbandry (Zhang et al.,2013; Liu et al., 2013; Lee et al., 2015; Farzaneh et al., 2018; Wang et al., 2019 ).

The methods of culturing chick embryo in windowed eggs provided an easy way for genetic manipulations of the embryo (Farzaneh et al., 2018). The use of eggs windowing technology can be illustrated in Figure 2. Two methods including surrogate eggshell and eggshell windowing were reported frequently (Farzaneh et al.,2018; Wang et 


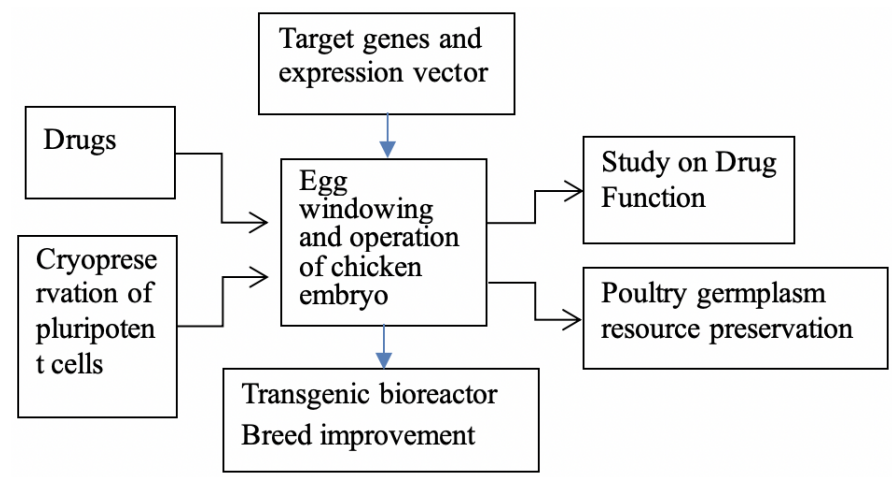

Figure 2 - Application of Egg Windowing Technology.

al., 2019. Although the surrogate eggshell method (Borwompinyo et al., 2005; Liu et al., 2012; Farzaneh et al., 2018) provides the advantages of microinjection, hatchability and observation of embryonic development, its complicated operation, high technical requirements, high cost, and susceptibility to contamination made it unfit for large-scale egg experiments needed for transgenic chickens.

In contrast, the eggshell windowing method was simple and easy to operate. It included windowing on the blunt end, the equatorial plane, and the sharp end of the eggshell. Windowing on the egg equatorial plane has been a common method for making chicken chimeras because it is the closest place to operate the embryo. However, there were many methods for cutting and sealing the window, and the reported results were conflicting.

Many factors could affect the efficiency of transgenic chicken production, egg windowing was one of the major concerns (Andacht et al., 2004; Wang et al., 2019). However, different sealing methods will give different results even with the same windowing methods. In addition, there were some other factors affecting the hatchability of windowed eggs such as air cell and injection. Andacht (et al., 2004) reported that the egg hatchability was severely decreased when air was injected into it. Naito (et al.,1991) reported that the survival rate of chick embryos was inversely proportional to the injection volume. It was also reported that there were significant differences when the different volume was injected into the windowed eggs (Lee et al., 2007 and 2013).

So far, there is no unified standard for the eggshell windowing and injection techniques. The patent of simplifying eggshell windowing methods was used, and the effects on different methods of sealing, air cell recovery, and injection volume on the hatchability of windowed egg were investigated in this study.

\section{MATERIALS AND METHODS}

\section{Experimental animals}

Fertilized White Leghorn (WL) and Black Silkise (BS) chicken eggs were collected from the animal house at the Hunan Institute of Animal and Veterinary Science, China. WL and BS chickens were fed in a standard management program. The procedures used for animal management, reproduction, and embryo manipulation followed the standard operating protocols of our laboratory.

\section{Experiment 1, the Sealing materials screening for the Windowed eggs}

The fresh fertilized chicken eggs were collected and stored at $16^{\circ} \mathrm{C}$ to $18{ }^{\circ} \mathrm{C}$. The eggs were sterilized by $75 \%$ alcohol and then horizontally placed on an egg tray for about $40 \mathrm{~min}$ at room temperature. The methods of eggshell windowing were done as the patent (ZL200710034253.0). Briefly, the windows were made by a special cutter (Miniature electric grinder, Asoyoga ASJ-8, Fig. 1-A) and a triangle groove with a side length of $6 \mathrm{~mm}$ was cut on the equatorial plane of the eggshell without destroying the inner shell membrane and the width of one groove was double as the other two (Fig. 1-B) . A $2 \mathrm{~mm}^{2}$ double sticky tape was pasted on the shell near the widest groove (Fig. 1-C). The egg was placed horizontally for 10 minutes with window upward. The inner shell membrane of the two narrow grooves were cut by scalpel, then the shell slice was turned over by the blade of scalpel (Fig. 1-C) and sticked to the tape (Fig.1-D). Most of time, the embryos could be seen and easy to be injected through the window.

The shell slices would turn back to cover the window along the grooves automatically when they were stripped from the sticky tape, and the windows were sealed using the following four methods respectively: 1) "SP+IG", straw powder (SP) (FHK, Japan) was sprinkled evenly on the groove, then one or two drops of instant glue (IG) (502, super glue, China) were dropped spreading along the whole grooves; 2) The yellow sticky paper tape (3M 244, Scotch Brand Tape, SBT) of side $1.2 \times 1.5 \mathrm{~cm}$ was pasted, centering on the cut area and smoothed with a scalpel. 3) The medical sterile sticky tape (SST) of side $1.2 \times 1.5 \mathrm{~cm}$ (SteriStripTM, American ) was pasted as the same method described previously. 4) "SST+IG", SST was pasted firstly and IG was dropped with the same methods described previously.

After being sealed, the eggs were placed in the incubator (Xu Shen, China) and hatched at $37.5{ }^{\circ} \mathrm{C}$ 
Yan HF, Wang K, Tang MX, Feng XH, Trefil P, Qiu MZ, Jiang J

A

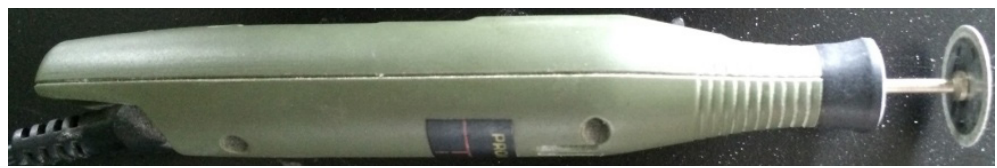

B

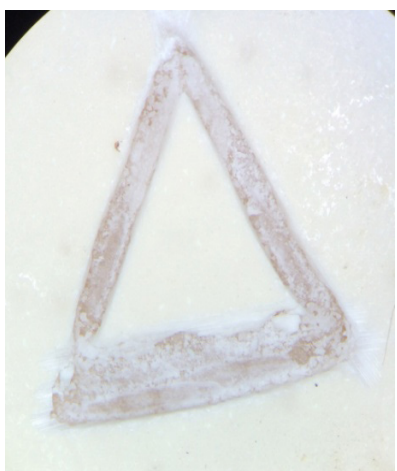

E

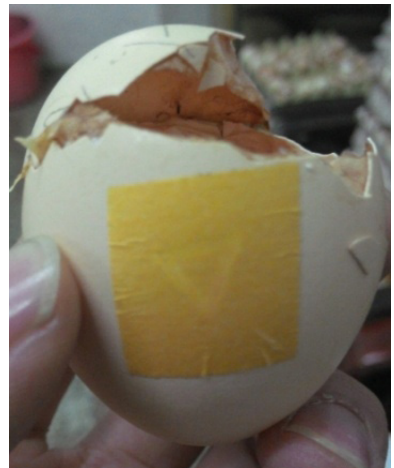

H

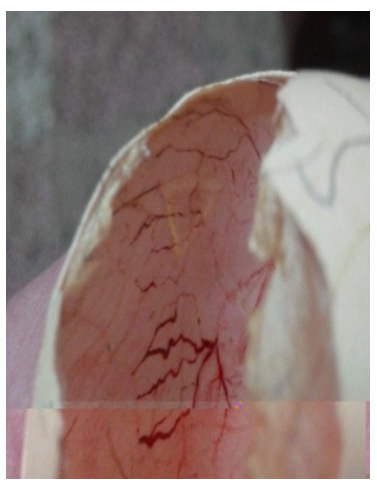

K

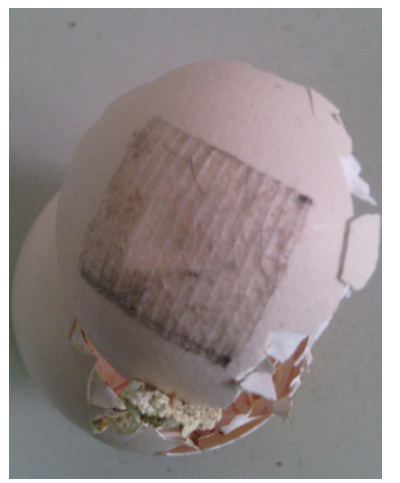

C

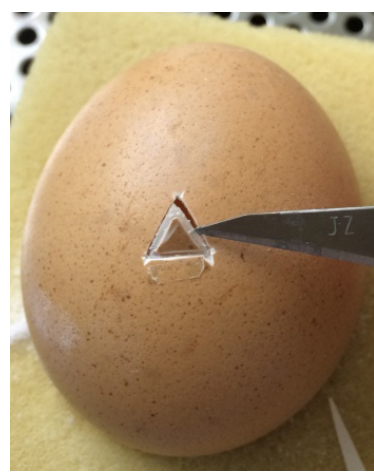

$\mathbf{F}$

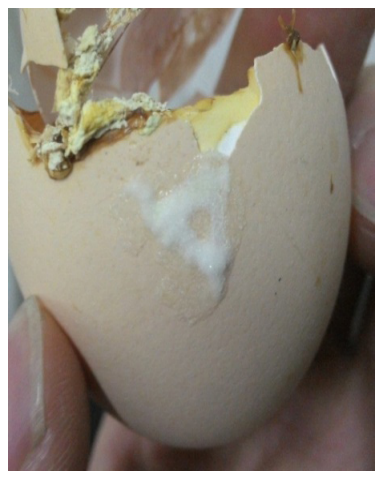

I

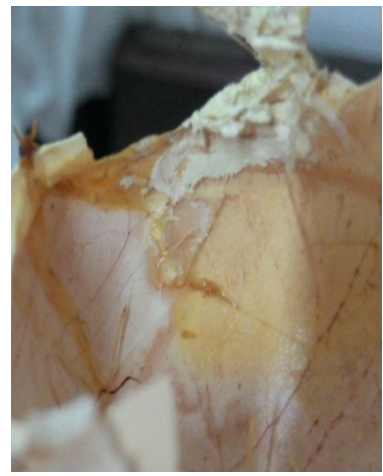

$\mathbf{L}$

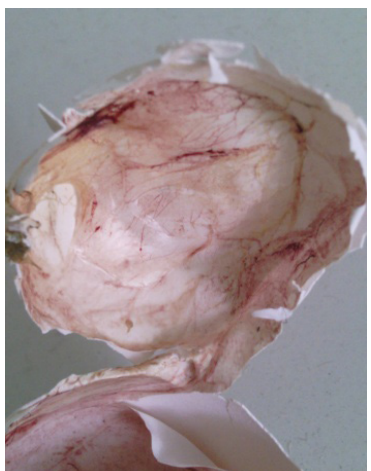

D

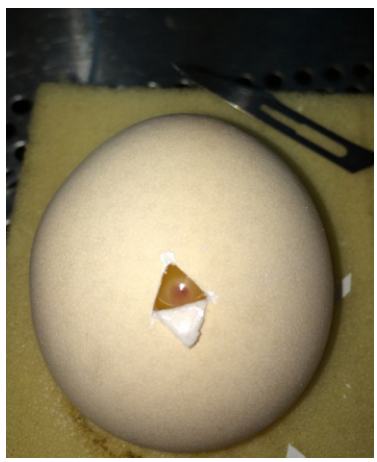

G

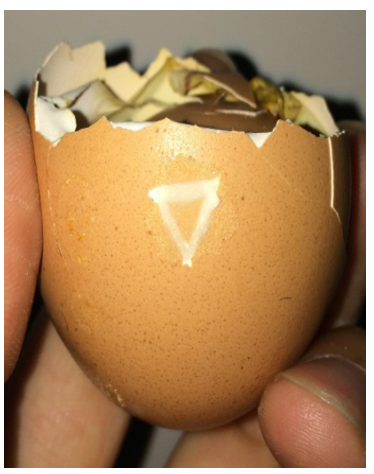

J

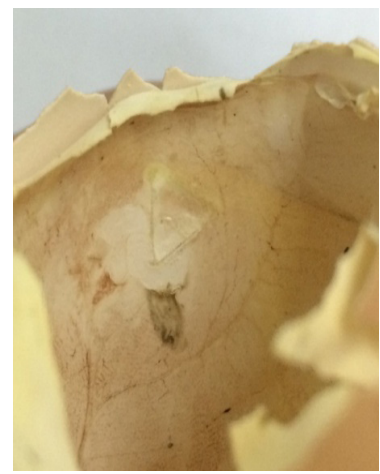

M

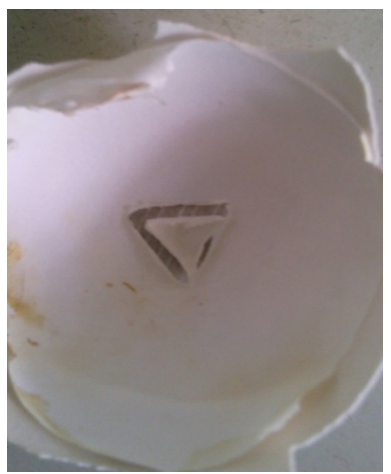

Figure 1 -A: Egg shell cutting tool. B: The triangle groove, only cutting the outer shell without destroying the inner shell membrane, the width of one side was two times the width of the other two. C: $2 \mathrm{~mm}^{2}$ double faced adhesive tape was glued beside the wider seam, and the inner membrane of the other two seams of the triangle were cut so that the small triangle cover can turn over along the wider seam. D: After opening the shell and injecting DMEM into the embryo, a red spot was apparent in the middle of the embryo. E: The outside part of the window sealed with the SBT( yellow sticky paper tape, 3M 244, Scotch Brand Tape). F: The outside part of the window sealed with "SP+IG" (Straw powder, FHK, Japan, was firstly sprinkled on the groove evenly, then one or two drops of instant glue, 502, super glue, China, were dropped spreading along the whole grooves. G: The window sealed with IG first, then sprinkled with SP. H: After hatching, the internal part of the window sealed with SBT. I: After hatching, the internal part of the window sealed with SP first, then dripped with IG. J: After hatching, the internal part of the window that was sealed firstly with the IG, then sprinkled with SP. K: The blunt end window sealed with SST (the medical sterile sticky tape with side of $1.2 \times 1.5 \mathrm{~cm}$, Steri-Strip ${ }^{\mathrm{TM}}$, American). L: After hatching, the punctured part of the inner membrane under the window being sealed with SST in the blunt end. M: After hatching and the inner membrane being removed, the internal part of the window which was sealed with SST in the blunt end. 
Yan HF, Wang K, Tang MX, Feng XH, Trefil P, Qiu MZ, Jiang J
Study on the Efficient Methods for Chicken Eggshell windowing and the Factors Affecting the Hatchability and $65 \% \mathrm{RH}$ (relative humidity) with $90^{\circ}$ turning angle every two hours. The eggs were put into the hatching tray and stopped turning after $18 \mathrm{~d}$, and were kept at the same temperature and humidity until $23 \mathrm{~d}$.

\section{Experiment 2, Air cell recovery for the windowed eggs}

A triangle window with a side length of $4 \mathrm{~mm}$ at the egg blunt end was cut, and the air cell together with the shell inner membrane was observed. Keeping the window opening, the eggs were placed horizontally and another triangle window was cut on the egg equatorial plane with a side length of $6 \mathrm{~mm}$ and the slice was turned over, and the embryo was found and recorded. Then the window on the equatorial plane was sealed and the egg was placed with blunt end upward. At that moment, the inner and outer membrane came to fuse together, the air cell disappeared and new bubbles appeared in the inner membrane. The needle of one syringe $(1 \mathrm{~mL})$ was stabbed into the inner membrane to drain air. The inner membrane collapsed and the air cell appeared again.

The two windows on one egg were sealed as the following three methods: 1) SBT-E (equatorial plane) and SST-B (blunt end); 2) SBT-E (equatorial plane) and SBT-B (blunt end); 3) SST-E (equatorial plane) and SST-B (blunt end). The sealed eggs were hatched by the methods described previously.

\section{Experiment 3, The adding order for using "SP+IG" and the laying direction immediately after being sealed}

According to the results screened previously, the eggs were windowed, sealed and laid by the following five methods: 1) "IG+SP+PH", the eggs were sealed with the "IG+SP" (Used IG firstly then SP) method and were immediately placed horizontally $(\mathrm{PH})$ on egg tray; 2) "IG+SP+ PBU" ,the eggs were sealed with "IG+SP" and were immediately placed with the blunt end upward (PBU); 3) "SP+IG+ PBU", the eggs were sealed with "SP+IG" (Used SP firstly then IG) and were immediately placed with "PBU"; 4) "SP+ $\mathrm{H}_{2} \mathrm{O}+$ PBU", the eggs were sealed with the methods of "SP", then one or two drops of $\mathrm{ddH}_{2} \mathrm{O}$ (containing $100 \mathrm{U} /$ $\mathrm{mL}$ benzylpenicillin (No.D0717, Biosharp, USA) and $100 \mu \mathrm{g} / \mathrm{mL}$ streptomycin (No. C1203, Biosharp, USA) ) were spread along the grooves, and were immediately placed with "PBU"; 5) "SST+PBU", the eggs were cut off just the surface of the shell without destroying the inner membrane, and were sealed with "SST", and then were immediately placed with "PBU". The eggs were hatched according to the same methods described previously.

\section{Experiment 4, the injection volume screening for the future chicken chimeras}

The glass capillaries (outer diameter was $0.16 \pm 0.06$ mm, Instrument Factory of Huaxi Medical University) were pulled on the flame of the alcohol lamp to make the microinjection glass needle. The tip of glass needle was cut with sandpaper, and the needle whose tip with regular fracture under 40 times dissecting microscope would be grinded into an inclined sharp plane with a angle of about 45 degrees using the needle grinder (Nashge Microforge). The simple microinjection system was composed of $1 \mathrm{~mL}$ syringe, No.18 needle, soft plastic tube, $5 \mathrm{~mm}$ length from the end of $200 \mathrm{uL}$ pipette tip and glass needle.

The eggs were windowed and the embryos were found through the window. $1 \mu \mathrm{L}, 3 \mu \mathrm{L}, 5 \mu \mathrm{L}, 10 \mu \mathrm{L}$ medium which contains DMEM solution (No. SH30022.01B, HyClone, China), 10\% FBS (Tianhang biological technology co., LTD, Zhejiang, China), $100 \mathrm{U} / \mathrm{mL}$ penicillin sodium (D0717, Biosharp) and $100 \mu \mathrm{g} / \mathrm{mL}$ streptomycin (C1203, Biosharp) were injected into the subgerminal cavity of the windowed eggs respectively. After being injected the eggs were sealed with the previously screened methods and hatched with the previous conditions.

\section{Eggs candling and air cells testing}

All eggs were candled on d 10, the unfertilized eggs and dead embryos were taken out. The hatching period extended to $\mathrm{d} 23$ because the egg embryonic development may be delayed after the windowing. All the eggs that did not hatched were candled and the air cells were recorded.

\section{Data Analyses}

Fertility $=$ (fertilized eggs/input eggs $) \times 100 \%$. Hatchability $=($ chicks/fertilized eggs $) \times 100 \%$. The data was analyzed by one-factor variance method of SAS 9.1 software.

\section{RESULTS}

\section{Comparison of different sealing materials}

Comparing the 7 sealing methods in Table 1, the hatchability of all windowed groups were significantly lower than the control groups $(p<0.01)$. The highest hatchability was $31.6 \%$ in the group "SP+IG". The hatchability of the SST group, SBT group and "SST+IG " group was $17.6 \%, 12.8 \%$ and $2.6 \%$ respectively.

The data of two windows (recovering air cell) was shown in Table 2. There was significant difference 
Table 1 - Hatchability of different sealing materials in egg groups with one window (mean $\pm S D ; n=3$ )

\begin{tabular}{lccccc}
\hline Groups & Total eggs & Fertility $(\%)^{\star *}$ & Alive embryos rate at 10 days $(\%)^{*}$ & Hatched egg rate $(\%)^{*}$ & Air cell rate $(\%)^{\star *}$ \\
\hline Control & 45 & $95.7 \pm 3.7$ & $95.2 \pm 4.1$ & $69.7 \pm 4.8^{\text {A }}$ & $97.9 \pm 3.6$ \\
SP+IG & 40 & $85.3 \pm 14.3$ & $85.2 \pm 5.5$ & $31.6 \pm 10.1^{\mathrm{B}}$ & $82.8 \pm 15.2$ \\
SST & 55 & $94.5 \pm 0.4$ & $78.9 \pm 2.0$ & $17.6 \pm 7.0^{\mathrm{C}}$ & $56.2 \pm 6.0^{2}$ \\
SBT & 84 & $83.5 \pm 4.8$ & $74.4 \pm 3.3$ & $12.8 \pm 3.8^{\mathrm{D}}$ & $36.8 \pm 6.2$ \\
SST+IG & 45 & $91.1 \pm 3.8$ & $78.4 \pm 14.0$ & $2.6 \pm 4.4^{\mathrm{E}}$ & --- \\
\hline
\end{tabular}

Note: The * and ** denote that the denominator was the fertilized eggs and the total eggs respectively. In the same column, different capital letters mean significant difference ( $p<$ 0.01). Control: Not windowed egg. The shell slices would turn back to cover the window along the grooves automatically when they were stripped from the sticky tape, and the windows were sealed by the following four methods respectively:

1) "SP+IG": Straw powder (FHK, Japan) was firstly sprinkled on the groove evenly, then one or two drops of instant glue (502, super glue, China) were dropped spreading along the whole grooves;

2) SST: The medical sterile sticky tape with side of $1.2 \times 1.5 \mathrm{~cm}$ (Steri-Strip ${ }^{\mathrm{TM}}$, American) was pasted in the middle of the cut area, and carefully smoothed with a scalpel.

3) SBT: The yellow sticky paper tape (3M 244, Scotch Brand Tape) with side of $1.2 \times 1.5 \mathrm{~cm}$ was pasted as the method of SST.

4) "SST+IG": SST was pasted firstly by the above method and then IG was dropped along the edge of the SST.

In the experiment of the sealing method, all eggs were incubated with the blunt end upward in a conventional incubator at $37.5 \%$ and $65 \%$ relative humidity until being hatched, and the eggs were turned $90^{\circ}$ every 2 hours for $18 \mathrm{~d}$, then were placed in hatching baskets without turning until being hatched.

Table 2 - The air cell rate and hatchability in egg groups with two windows (mean $\pm \mathrm{SD} ; \mathrm{n}=3$ ).

\begin{tabular}{lccccc}
\hline Groups & Total eggs & Fertility $(\%)^{* *}$ & Alive embryos rate at 10 days $(\%)^{*}$ & Hatched egg rate $(\%)^{*}$ & Air cell rate $(\%)^{* *}$ \\
\hline Control & 45 & $95.7 \pm 3.8$ & $95.5 \pm 3.9$ & $69.8 \pm 1.4^{\mathrm{A}}$ & $98.0 \pm 3.4$ \\
SBT-E and SST-B & 24 & $92.1 \pm 6.9$ & $67.9 \pm 9.4$ & $17.9 \pm 6.2^{\mathrm{B}}$ & $84.7 \pm 16.8$ \\
SBT-E and SBT-B & 74 & $92.1 \pm 3.3$ & $78.0 \pm 3.3$ & $11.7 \pm 2.0^{\mathrm{C}}$ & $84.7 \pm 11.5$ \\
SST-E and SST-B & 20 & $95.2 \pm 8.2$ & $68.3 \pm 2.7$ & $5.6 \pm 9.6^{\mathrm{D}}$ & $65.1 \pm 7.3$ \\
\hline
\end{tabular}

Note: The * and ** denote the same meaning as table 1. SBT-E and SST-B: One window on equatorial plane covered with SBT and another one on blunt end covered with SST; SBT-E and SBT-B: One window on equatorial plane covered with SBT and another one on blunt end covered with SBT; SST-E and SST-B: one window on equatorial plane covered with SST and another one on blunt end covered with SST. The sealed eggs were hatched by the previously described methods as table 1.

among each group of chambers recovered $(p<0.01)$. The highest hatchability was $17.9 \%$ in the group of "SBT-E and SST-B". The hatchability was $11.7 \%$ and $5.6 \%$ in the groups of "SBT-E and SBT-B" and "SST-E and SST-B" respectively. The external and internal part of the window was shown in Fig. 1-E to J.

\section{Relationship between the air cell rate and hatchability}

We found that there were all air cells in the hatched eggs, so we only needed to check the air cells of the unhatched eggs and get the air cell rate of windowed eggs. The average air cell rate of the groups with unrecovered air cell groups was $45.1 \%$. The correlation coefficient of air cell rate and hatchability was 0.91 (Table 1).

There was high-positive relationship between the rate of air cell and hatchability. The average air cell rate of the groups with recovered air cell was $80.5 \%$, and the air cell rate in recovered groups was always higher than that of unrecovered group. The air cell rate of the "SST-E and SST-B" group was the lowest. The blunt end window sealed with SST was shown in Fig.1-K. After hatching, there was still a pinhole unhealed in the inner membrane under the window, which was sealed with SST in the blunt end (Fig.1-L).

Table 3 - Hatching results of the different adding order of the sealing materials and different laying position of windowed eggs (mean $\pm \mathrm{SD} ; \mathrm{n}=3$ ).

\begin{tabular}{lcccc}
\hline Groups & Total eggs & Fertility (\%)** & $\begin{array}{c}\text { Alive embryo rate at 10 days } \\
(\%)^{*}\end{array}$ & Hatchability $(\%)^{*}$ \\
\hline Control & 66 & $97.0 \pm 2.6$ & $97.0 \pm 2.6$ & $78.3 \pm 8.9^{\mathrm{B}}$ \\
SST (shell membrane not destroyed) & 20 & $84.7 \pm 2.4$ & $88.6 \pm 10.3$ & $83.8 \pm 14.7^{\mathrm{A}}$ \\
IG+SP (blunt end upward) & 77 & $95.9 \pm 4.2$ & $96.1 \pm 3.6$ & $71.3 \pm 3.4^{\mathrm{C}}$ \\
SP+IG (blunt end upward) & 90 & $94.3 \pm 2.1$ & $88.0 \pm 1.3$ & $48.7 \pm 4.7^{\mathrm{D}}$ \\
SP+IG (equatorial plane upward) & 90 & $88.2 \pm 4.3$ & $83.8 \pm 1.9$ & $32.5 \pm 3.9^{\mathrm{F}}$ \\
SP+ ddH ${ }_{2} \mathrm{O}$ (blunt end upward) & 104 & $85.6 \pm 5.3$ & $88.0 \pm 5.7$ & $35.3 \pm 1.0^{\mathrm{E}}$ \\
\hline
\end{tabular}

Note: The * and ${ }^{* *}$ denote that the denominator is the fertilized eggs and the total eggs respectively; In the same column, different capital letters mean significant difference ( $\left.p<0.01\right)$. The other information is the same as the table 1. 


\section{The comparison of different adding orders and different placing methods after sealing}

The results (Table 3 ) showed that there was significant difference among each group $(p<0.01)$. The hatchability of the group without breaking the inner membrane was significantly highest one $(p<0.01)$. Among the membrane being cut groups, the hatchability of the group "IG+SP" (sealed with IG first and then SP) was $71.3 \%$ and significantly higher than the group "SP $+I G$ " (sealed with SP first and then $I G)$, and it was also significantly higher than any other group $(p<0.01)$. The hatchability of the group placed blunt end upward was significantly higher than that of the group placed horizontally with the same sealing method $(p<0.01)$. The hatchability of the group, which placed blunt end upward after being sealed with SP first and one or two drops of $\mathrm{dd}_{2} \mathrm{O}$ dropped, was significantly lower than any other groups $(p<0.01)$.

\section{Comparison of different injection volumes}

As the result was shown in Table 4 (the denominator was the total eggs), the hatchability of every treatment group was significantly lower than that of the control group $(p<0.01)$. The highest hatchability among the treatment groups was $69.1 \%$ in the group without injection, and there was significantly difference with the other groups. The hatchability were $48.9 \%, 34.7 \%$, $29.7 \%$ and $10.3 \%$ of the group with $1,3,5$ and $10 \mu \mathrm{L}$ injection volumes respectively. The highest hatchability was in the group of $1 \mu \mathrm{L}$ and it was significantly higher than any other group $(p<0.01)$. The hatchability of the $10 \mu \mathrm{L}$ group was the lowest, and it was significantly lower than any other group $(p<0.01)$. The difference between $3 \mu \mathrm{L}$ and $5 \mu \mathrm{L}$ group was significant $(p<0.05)$.

\section{DISCUSSION}

\section{The strategy of eggshell cutting for the window}

The strategy of eggs windowing includes finding a cutter, cutting mode and cutting position. There are three windowing positions on the eggshells, such as the blunt end, the equatorial plane, and the sharp end of the eggshell.

When the eggs were windowed on the blunt end, the air cell was destroyed and it was not easy to inject the embryos due to its far distance to the embryo. Windowing at the sharp end was widely used for 14-day and later-stage embryos (Yamamoto et al., 2007; Kang et al., 2008; Nakamura et al., 2010), but for X-stage embryos, the hatchability was low, and it was also a long distance to operate the embryo. The eggs were incubated for 48-52 hours with a window in a diameter of $20 \mathrm{~mm}$ in the sharp end and the hatchability was $68.2 \%$ (Nakamura et al., 2010). The transgenic broilers were produced by eggs, which were incubated for 55-56 hours and windowed on blunt end (with air cell, located at the blunt end and between the two layers of the egg shell) and sharp end (non-air cell), and the hatchability was $70.0 \%$ vs. $53.3 \%(p<0.05)$ (Wang et al., 2019). The hatchability on blunt end and equatorial plane of Stage $X$ embryos was $61.0 \%$ and $45.0 \%$ respectively (Yan et al., 2008). Zhang (et al., 2013) found that the hatchability was $31.67 \%$ and $25.00 \%$, when the newly laid eggs were windowed on the equatorial plane and blunt end respectively, but there were no chicks hatched when the method of surrogate eggshells was used.

These reported methods were usually used in cutting the triangle eggshell slice off and transferring them to a moisturizing environment. The eggshell slice was transferred to cover the window after the embryo operation had been finished. It was difficult to find the original direction of the eggshell slice fit for the window when it was put back, most of the time it was very easy to fall into the window and the whole work was failed. In many cases, there were no detailed descriptions of how to open the windows, how to cut the windows and how to seal them. The slices of the window were destroyed and the window was sealed with parafilm or hot glue (Heo et al.,2012; Lee et al., 2013; Zhang et al., 2013; Farzaneh et al., 2018; Wang et al., 2019).

Table 4 - Survival rate and hatchability of the different injection volume (mean $\pm S D ; n=3$ ).

\begin{tabular}{lcccc}
\hline Groups & Total eggs & Quickly died rate (\%) & Alive embryo rate at 10 days (\%) & Hatchability (\%) \\
\hline Control & 34 & $7.2 \pm 7.7$ & $83.6 \pm 9.0$ & $79.4 \pm 2.0^{\mathrm{A}}$ \\
No injection & 77 & $3.8 \pm 3.5$ & $92.3 \pm 2.1$ & $69.1 \pm 4.1^{\mathrm{B}}$ \\
$1 \mu \mathrm{L}$ & 41 & $19.6 \pm 0.7$ & $74.9 \pm 5.8$ & $48.9 \pm 1.9^{\mathrm{C}}$ \\
$3 \mu \mathrm{L}$ & 38 & $31.6 \pm 4.6$ & $60.2 \pm 5.0$ & $34.7 \pm 3.0^{\mathrm{Da}}$ \\
$5 \mu \mathrm{L}$ & 34 & $38.2 \pm 6.4$ & $53.4 \pm 3.0$ & $29.7 \pm 3.2^{\mathrm{Db}}$ \\
$10 \mu \mathrm{L}$ & 39 & $47.9 \pm 8.6$ & $17.1 \pm 6.2$ & $10.3 \pm 3.8^{\mathrm{E}}$ \\
\hline
\end{tabular}

Note: The denominator is the total eggs. In the same column, different capital letters mean significant difference $(p<0.01)$, and the different lower case letters mean significant difference $(p<0.05)$. 
In this paper, it indicated that there were many advantages of the equatorial plane windowing, such as easily finding the embryo and the window close to the embryo, which was efficient and convenient for injection. Especially, the invented in-situ cutting and the eggshell slice automatically recovering methods in this paper, could avoid the great damage to the integrity of the eggshell, and the efficiency for cutting the eggshell, finding and injecting the embryo, and sealing the window for more than 30 eggs per hour per person. It also showed that 40 min was enough to find the embryo when eggs were put in a horizontal direction, and the probability of finding embryo did not increase obviously as time increased. For the eggshell cutter, it was difficult to get according to the reported paper. It was a miniature electric grinder, also known as an engraving machine, engraving pen. It was easy to buy online and could greatly improve the efficiency of egg shell cutting.

\section{The strategy of eggshell window recovery}

At present, the most common sealing materials are paraffin, fresh shell membrane, and parafilm simultaneous with hot glue (Speksnijder et al., 2000; Zhang et al., 2013; Lee et al., 2013). Based on the same opening position, the hatchability obtained by these materials were $48.9 \%, 35.6 \%, 35 \%$, and $60 \%$ respectively. It indicated that the sealing materials could have a significant impact on the hatchability.

We compared the hatchability for the seven types of sealing methods and found that the best way was the straw powder together with instant glue $(\mathrm{SP}+\mathrm{IG})$. But the overall hatchability was still low. Albumen is a transparent colloidal substance consisting of proteins, wrapped around the yolk. SP is polyethylene dilute alcohol powder, can be solidified when wetted by water. SP aqueous solution is easy to form film, which is colorless, transparent and good mechanical strength. It can be mixed with starch, gum, cellulose derivatives and various surfactants and has good stability. IG is a colorless transparent liquid with good fluidity, and easy to volatilize. When IG comes to water, it is polymerized and cured. It was similar to the hot glue method (Andacht et al., 2004, Lee et al., 2013) because the working time of hot glue was $15 \mathrm{~s}$ and the sealing process was also fast. It shows that the shorter of the sealing time the better result of the hatchability. Even if drilling a smaller $(3 \times 3 \mathrm{~mm})$ window and using PBS to eliminate air inside the window, the hatchability was $45.3 \%$ and $60.0 \%$, which was lower than the hatchability obtained by this study. In addition, the hot glue method needed a professional tool and was difficult to do.

At the present time, opening on the equatorial plane and after being sealed, the eggs must be placed horizontally with the window upward, and the albumen should not touch the window as far as possible. Only by keeping the sealing area dry for some time, can the sealed eggs be placed with blunt end upward. It might be harmful to the embryo if it contacting the window. In terms of the complexity of the physical and chemical properties of the albumen, IG and SP, the adding order of the IG and SP, and the placing methods of the eggs may have interaction effects.

We found that the different orders of adding the sealing materials and the laying positions after sealing could significantly affect the hatchability. The order of dropping IG first and then sprinkling SP was better than the opposite order. Compared the internal appearance of the two methods (Fig. 1-I,1-J), the egg with IG first was smoother than the other. When SP were sprinkled first, it probably entered the seal and contacted the albumen (sometimes even the embryo). Embryo always floats above the yolk and if the windowed egg is put with window on the top, embryo will be close to the window. If the eggs are placed with blunt end upward immediately after sealing, it can keep the embryo away from the window and can not make the potential damage of the sealing material. This viewpoint was confirmed by the results (Table 4). Without the improved method previously, the eggs can not be placed blunt end upward immediately after sealing, because the water will permeate into the seals and make the sealing materials out of action.

It indicated that the hatchability and flatness of the inner part of the window was the best by using the glue before powder and laying blunt end upward immediately after being sealed. First, thanks to our "hinged" windowing method, the "cutslice" only needs to peel off the adhesive tape, and it can automatically cover the window. The covering is very simple and fast. Secondly, the slice has a certain bearing capacity and it is not easy to fall into the window. Thirdly, the slice fits the window very well by the in situ covering method, leaving very small seams, with little albumen in the seams and less protein on the outer surface of the slice. When IG is used first, the glue which plays a leading role can basically seal the seams because of its advantages such as less dosage, water use, quick drying and bridging of small seams. When SP being added later in the seam, it needs water to solidify, there will be the remaining egg white, water, or IG in the seam, and the SP will play a second reinforcing role 
for the window sealing, even when the eggs was put blunt end upward immediately after being sealed. On the contrary, if being used firstly the SP will mix with the water and mainly plays a blocking role (difficult to play a bridge role) in the seal. In addition, the powder is easy to fall into the hole and collapse the cutting slice when it was used before IG.

As for the placing method, the embryo always floats to the blunt end when it is placed blunt end upward and does not contact the window, which was better for the embryo development.

Therefore, the sealed window was airtight and hardly leaked till $d 28$ of incubation, and the appearance of internal membrane was smooth after hatching (Fig. 1-J). If sealed with SBT and SST, it was easy out of action when the tape contacted the albumen. In many cases, the albumen leaked out of the sealed window, and the bacteria had chance to enter eggs. The hatchability of all eggs sealed with the tape was low, maybe due to these reasons. The hatchability of windowed eggs got to $68.8 \%$ when they were sealed by using IG first then SP, and laid blunt end upward immediately. So, not only the details of the operation were improved, but also the hatchability was stabilized and enhanced in this paper. It took only $30 \mathrm{~s}$ to seal, and the efficiency for windowing, injecting, and recovering were the highest at the present time ( more than 30 eggs per hour per person).

\section{The air cell recovery of the windowed egg}

There is an air cell between the inner and the outer membranes at the blunt end. After windowing, new bubbles are created in the albumen, and the inner atmospheric pressure balance is broken, finally the air cell shrinks or even disappears. Andacht (et al.,2004) tried to inject air into the albumen to produce new bubbles in egg, and the hatchability was reduced from $63.2 \%$ to $15.7 \%$. So it indicated that the hatchability was significantly influenced by bubbles. It also revealed that due to the low density of air cell and embryos, they always adhere to each other and float on the top part of the egg. Even if the eggs rotate, the trend does not change, thus preventing the formation of stable allantoic membrane along the shell membrane, and affecting the growth of embryos (Andacht et al., 2004). PBS was added into the egg window before being sealing, to eliminate bubbles, but the hatchability was uncertain (Speksnijder et al., 2000, Wang et al.,2015, Yan et al.,2008).

In fact, adding PBS can eliminate bubbles but the shrinking or disappearing air cell can not be recovered. We find that the time from opening to sealing and the swing range of eggs after windowing, determine the shape of the air cell after sealing. The shorter the time from windowing to sealing and the smaller the swing range after the egg windowing were, the smaller the air cells after sealing were. If on the contrary operating, the air cells would shrink or even disappear. It was the first time to try to make two windows for recovering the air cell of the windowed eggs. The results indicated that there was a positive correlation between air cell rate and hatchability. A high rate of air cell was observed in the group of IG and SP, which might be the reason of shorter sealing time. Perhaps the sealing tape needs permeability for the eggs to exchange air during hatching, so they should be airtight on equatorial plane and should be in good permeability at blunt end. We found that bubbles would appear when permeability tape was sealed on equatorial plane after several days of hatching, even with the air cell appearing. A permeability tape sealed at blunt end led to higher hatchability, probably due to the previously reason.

\section{Other factors affecting the hatchability of windowed eggs}

The hatchability was significantly decreased after injection and there was an inverse proportion between embryo survival rates and injection volumes. The hatchability of newly laid eggs were $8.6 \%$ and $40.3 \%$ when the volumes of the cell suspension injected were 3-5 $\mu \mathrm{L}$ and $1 \mu \mathrm{L}$, respectively (Naito et al.,1991). A 4×4 $\mathrm{mm}^{2}$-sized window was made and the hatchability was $70.1 \%, 42.7 \%$ and $8.3 \%$ for the egg groups of windowed, medium injected and retrovirus vector injected respectively (Lee et al.,2007). It was reported that $0.5 \mu \mathrm{L}$ cell suspension was enough but it was too small to see and control and the experimental repeatability was very poor (Naito et al.,1991). The effects of different injection volumes on hatchability were compared in this study and it indicated that the windowing decreased hatchability at least by 10.3 percentage points, and injecting decreased at least by 20.2 percentage points. The highest hatchability in this paper was $48.9 \%$ in the group with $1 \mu \mathrm{L}$ DMEM, and it was the highest in the reported paper we found. Little difference of hatchability was found between the $3 \mu \mathrm{L}$ and the $5 \mu \mathrm{L}$, but the hatchability decreased dramatically to $10.3 \%$ in the group with $10 \mu \mathrm{L}$. The diameter of the injection needle in this experiment was large and we made it by hand and it was cheap and easy to do. The hatchability will be higher if the injection needle is thinner. In addition, the hatchability of the control group was low $(79.4 \%)$, perhaps the main reason was the quality of the eggs. 
Considering the choice of the embryonic stage for the injection, it was reported that chicken embryos that hatched 3 days were the most suitable for injection, and the hatchability was 10 percentage points higher than that of the $X$ stage embryo (Dunn et al., 1997). The highest hatchability was $42.7 \%$ for injecting medium into X-stage embryos (Lee et al., 2007, Heo et al.,2012). However, when injected 14-17d stage embryos, the highest hatchability was $68.2 \%$ (Yamamoto et al., 2007; Kang et al., 2008; Nakamura et al., 2010). It indicated that the older the receptor embryonic stage was, the higher the hatchability was. However, the differentiation of the embryo will strengthen as the embryo develops, so the chance of forming chimera will decrease. Therefore, we must pay attention to choose the right embryonic stage for the injection.

To sum up, a systematic, simple and efficient study on eggshell windowing and injecting for chicken embryo was done, and the efficiency was the highest at the present time ( more than 30 eggs per hour per person). These techniques may be broadly used in the fields of avian transgenesis, genetic resources preservation, and even the embryonic development model of human medicine.

\section{REFERENCES}

Andacht T, Hu W, Ivarie R. Rapid and improvement method for windowing eggs accessing the stage $X$ chicken embryo. Molecular Reproduction and Development 2004; 69(1): 31-34.

Borwompinyo S, Brake J, Mozdziak PE, Petitte JN. Culture of chicken embryos in surrogate eggshells. Poultry Science 2005; 84(9): 14771482.

Dunn BE, Boone MA. Growth and mineral content of cultured chick embryos. Poultry Science 1977; 56(2):662-672.

Farzaneh M, Attari F, Khoshnam SE. The method of chicken whole embryo culture using the eggshell windowing, surrogate eggshell and ex ovo culture system.British Poultry Science 2018; 59(2): 240-244.

Heo YT, Lee SH, Kim T, Kim NH, Lee HT. Production of somatic chimera chicks by injection of bone marrow cell into recipient blastoderms. Journal of Reproduction and Development 2012; 58(3):316-322.
Kang SJ, Choi JW, Kim SY, Park KJ, Kim TM, Lee YM, Kim H, Lim JM, Han JY. Reproduction of wild birds via interspecies germ cell transplantation. Biology of Reproduction 2008;79(5): 931-937.

Lee $\mathrm{HJ}$, Lee HC, Han JY. Germline modification and engineering in avian species. Molecules and cells 2015; 38(9):743-749.

Lee SH, Gupta MK, Han DW, Han SY, Uhm SJ, Kim T, Lee HT. Development of transgenic chickens expressing human parathormone under the control of a ubiquitous promoter by using a retrovirus vector system. Poultry science 2007; 86(10): 2221-2227.

Lee SH, Gupta MK, Ho YT, Kim T, Lee HT. Transgenic chickens expressing human urokinase-type plasminogen activator. Poultry science 2013; 92(9): 2396- 2403.

Liu C, Zu J, Baskar V, Wernery U, Chang IK. Culture of chicken embryo in interspecific surrogate albumen. Poultry science 2012; 91(11):28662871.

Liu XJ, Li N, Hu XX, Zhang RQ, Li Y, Cao DN, Liu TX, Zhang YQ, Liu XF. Efficient production of transgenic chickens based on piggyBac. Transgenic Research 2013; 22(2):417-423.

Naito M, Watanabe M, Kinutani M, Nirasawa K, Oishi T. Production of quail-chick chimaeras by blastoderm cell transfer. British Poultry Science 1991; 32(1):79-86.

Nakamura Y, Usui F, Ono T, Takeda K, Nirasawa K, Kagami H, Tagami T. Germline replacement by transfer of primordial germ cells into partially sterilized embryos in the chicken. Biology of Reproduction 2010; 83(1), 130-137.

Speksnijder G, Ivarie R. A modified method of shell windowing for producing somatic or germ line chimeras in fertilized chicken eggs. Poultry Science 2000; 9(10): 1430-1433.

Stern CD. The chick: a great model system becomes even greater. Developmental Cell 2005; 8(1): 9-17.

Wang ZB, Du ZQ, Wei N. Production of transgenic broilers by non-viral vectors via optimizing eggshell windowing and screening transgenic roosters. Poultry science $2019 ; 98: 430-439$.

Yamamoto Y, Usui F, Nakamura Y, Ito Y, Tagami T, Nirasawa K, Matsubara Y, Ono $\mathrm{T}$, Kagami $\mathrm{H}$. A novel method to isolate primordial germ cells and its use for the generation of germline chimeras in chicken. Biology of Reproduction 2007; 77(1):115-119.

Yan HF, Wen P, Deng Y. A study on fertile eggshell windowing and its effect on embryo development. Acta Laser Biology Sinica 2008; 17(6):834839 .

Zhang YN, Yang HY, Zhang ZT, Shi QQ, Wang D, Zheng MM, Li BC, Song JZ. Isolation of chicken embryonic stem cell and preparation of chicken chimeric model. Molecular Biology Reports 2013; 40(3):2149-2156. 
